\title{
FORMING PERSONA THROUGH METRICS: CAN WE THINK FREELY IN THE SHADOW OF OUR DATA?
}

\author{
SUnEEL JETHANI AND NADINE RAYDAN
}

\begin{abstract}
The use of biosensors in wearable activity tracking devices to measure, record and share many aspects of life has been received with great enthusiasm for their potential to enhance conceptions of self through measuring variables relating to an individual's health and productivity. In 2012, readers of the Economist were introduced to the idea of using numbers on oneself in the same way that charting progress towards a goal is commonplace in business. A new culture of selfimprovement termed 'self-tracking' was beginning to gain currency. At the time that self-tracking was becoming mainstream it was estimated that the mobile health and diagnostics market was worth approximately US\$640 million, which would grow to US\$8.03 billion by 2019. In popular culture (news items and blogs), people who track their activity using technology are seen as heroic figures who are insightful, actualised, virtuous, and in control. Experimentation (trial and error), active intervention (a health kick, diet or detox), preventative self monitoring (blood pressure, glucose levels, heart rate) or the conscious foregrounding of habits (hydration, caffeine intake, counting steps) are constructed as rewarding. This can be seen as a manifestation of applying management principles to personal healthcare, and by extension the practice of applying an exacting science to the management of everyday life. Focusing on three current consumer technologies: Fitbit, Jawbone UP and Apple's HealthKit application and developer platform, we argue that using such devices fixes individuals to symbolic discourses, permissions, limits, and thresholds, which prefigure and enclose energies directed towards the formation of self-knowledge and conception of selfhood.
\end{abstract}

\section{KEY WORDS}

Quantified self; wearables; self-tracking; personal metrics; technological affordances

\section{INTRODUCTION}

Human understandings of the relation between bodily states and their personification have passed through various technological epochs following different paths of mediation, abstraction, and performance. Each of these eras has correlated strongly with dominant modes (enlightenment reasoning, industrial labour, immaterial and social labour) and means of production such as the type of instrumentation available, or being developed, at the time (i.e. 
oral, text-based, image-based, and then digitised). The instrumentation arising out of each technological epoch-for instance, the popularisation of body knowledge associated with public anatomical dissections of cadavers and the exchange of anatomical information via drawings and printed atlases during the 17th and 18th centuries-is a key mediator of knowledge deployed conceptualising the self, and performing oneself to others. Later technologies such as stethoscopes, sphygmomanometer (blood pressure measuring instruments), x-rays and other nuclear imaging, ultrasound and magnetic resonance imaging, and most recently bioinformatics, gene sequencing, and advanced biometrics, all produced new conceptions and metaphors for the cognition of bodily materiality.

More recently, Gary Wolf of Wired magazine, a co-founder of the "quantified self" movement, has asserted in The New York Times that in the "cozy confines of personal life" it was rare to yield the "power of numbers" and that "[a] journal was respectable. A spreadsheet was creepy". Wolf, in the same article states:

Two years ago, as I noticed that the daily habits of millions of people were starting to edge uncannily close to the experiments of the most extreme experimenters, I started a website called the Quantified Self with my colleague Kevin Kelly. We began holding regular meetings for people running interesting personal data projects. I had recently written a long article about a trend among Silicon Valley types who time their days in increments as small as two minutes, and I suspected that the self-tracking explosion was simply the logical outcome of this obsession with efficiency. We use numbers when we want to tune up a car, analyse a chemical reaction, predict the outcome of an election. We use numbers to optimise an assembly line. Why not use numbers on ourselves?

The Quantified Self movement was founded by Gary Wolf, along with Kevin Kelly, in 2007 at the height of Web 2.0 optimism. The pair have stated that they named the movement after observing the increasing popularity of tracking and logging personal data on variables including heart rate, physical activity, or sleep among a host of others, using various smartphone applications. As these practices gained purchase among various groups over the world this led in turn to the development of the quantified self 'meet up' where participants would share findings of their self-experimentation. These "show and tell" sessions revolved around a simple three question formula: What did you do? How did you do it? What did you learn? However, a question remains: how critical can life-auditing be with methodologies and technologies available to self-trackers at present?

There are four key developments that have contributed to the currency of the quantified self. First is the shrinking in size of the sensors themselves. Second is ubiquity of sensory components through their inclusion in smartphones and wearable computing. Third, social networking produced and normalised a certain social affordance of sharing. Fourthly, cheaper remote storage and cloud computing coupled with the computational power to process so called "big data" made it possible and acceptable to collect and contribute personal data to remote server locations.

In this paper, we inspect the various ways in which persona is formed through the use of sensor-based, data driven wearable devices such as the Fitbit Jawbone Up, and the Apple Watch among a growing number of others can be situated within a broader trajectory of bodymediation technology. We then discuss the epistemological and therapeutic role of self-tracking devices as part of contemporary networked and technocratic cultures of innovation and selfmanagement. We go on to look towards ways by which personification through quantification and metrics commodifies not only the performative presentation of self, but also the routes to 
self-knowledge on which persona formation is based. Two distinct yet mutually constitutive bio-political domains of persona formation flow through practices of self-tracking. One is the grounding of self-quantifier archetypes in cultures of auditing, archiving, and early adoption of technology. The second relates to data ontologies in which technologically garnered selfknowledge is made meaningful. We argue that, despite their novelty and the relative fidelity with which they can bring hidden aspects of the self to our attention, the production of intimate self-knowledge through sensor technology is unlikely to contribute to emancipatory forms of persona-formation.

In The Self-knower: a Hero Under Control, Robert Wiklund and Martina Eckert point out that society's preoccupation with knowledge of the self draws much of its gravitas from popular culture, lifestyle marketing and the ideological belief that by drawing attention to one's 'inner being' the hidden risks and potentials that factor in the pursuit of one's self-betterment can be addressed (v). This view comes from a long line of thinkers working within the field of clinical social psychology (see Allport, Jourard, Rogers, Maslow, Markus, Warsaw and Davis, cited in Wiklund and Eckert). In the contemporary moment, the pre-occupation with self-knowledge is perhaps most sharply observed among the growing number of people who, using a range of sensor-enabled, data driven and wearable devices, 'self-quantify' their lives. As a new user explains on the Quantified Self website:

I am new to "formal" self quantifying ... I've tracked information about myself for a long time, mostly on paper, but never thought of it in this context. Lately I've started to play around with apps and devices. It's all very confusing as there are so many of them. To start with, I'm focusing on logging what I eat. I have a sweet tooth and hope that by having to log every muffin and every piece of candy, I may be able to get control of it. I'm logging steps as well, hoping for the same motivational force [2014-Oct-20, 11:48]

This type of personal archival is received very differently from the work of Steve Mann whose experiments with wearable computational photography and counter surveillance resulted in a famous altercation in a McDonalds in Paris. There, an employee assaulted Mann trying to pull off the wearable computer vision system he had invented and worn for decades - essentially a prototypic device similar to Google Glass - and ejected him from the restaurant (wearcam.org). One reason for this shift in reception is a move away from the visual archival of life to the numerical representation of processes, which can be equated to one's vitality and this connects back to Wolf's claim that in the context of one's personal life, spreadsheets are "creepy."

However, many people are subjecting themselves to exactly such regimes of record keeping by attaching various sensor enabled wearable technologies to the body to track virtually any aspect of their life. A spreadsheet may still remain 'creepy' or too arduous to be an effectively sustainable form of self-knowledge production, but the ubiquity, unobtrusiveness, and seeming passivity of a lightweight, relatively discrete bracelet has proven to be less so. Large numbers of people across the world are using wearable technology to measure and quantify the body, hoping to answer questions such as: Why do I feel sluggish? How can I improve my health? Am I at risk? This seems to be a method of taking control of the production of knowledge about themselves, something that was once the exclusive domain of medical practitioners or folk knowledge.

Here, we critically explore persona-formation through the use of technology to produce a personal data repository intended to formulate a seemingly objective sense of the body and self. We argue that practices of technically mediated self-quantification are aligned to externally calibrated goals, thresholds, norms, and expectations which are framed by socialised 
representations of what is "healthy" or "productive". Further, we suggest these measures exhibit a complex relation of knowledge production that is interventionist by nature and selfdirected in the sense that the participant pays, and in some cases admits oneself to a community dedicated to achieving a similar task, essentially self-knowledge through data. Quantitative or measurement-based technology is deployed in the mining of the user's bodily states and behaviours, before banking this data and analysing it by a qualitative feedback mechanism consisting of algorithms and various visualisation tools. These tools are available to the user and/or others through a synching mechanism which provides real-time data via a web-based 'analytic' dashboard, along with the ability to share this data across social networks or with other applications, provided the user grants such permissions.

In what follows we engage with questions of how the production of self-knowledge is defined within such systems. How might the $n=1$ nature of self-enquiry, and its re-integration into the technical, ideological and institutional structures, give rise to the ontologies that make such data meaningful at the aggregate or population level $(n=a l l$, all available or all that can be inferred given the data available to the system).

We suggest that the relation between self-tracking and persona formation lies in the movement of practices of quantification and measurement at the peripheries of our lives to a more central epistemological locus. The feeding of data into a machine and its playback forms a rhetorically quantitative representation of self, which under the auspices of self-betterment, reflection on behaviours in real time, and learning new life strategies as a result, forms an intimate manufacture of persona which interpolates the subject as empowered through selfknowledge and a mastery of its technical systems of production. In the past, the production of self-knowledge at this level of intimacy depended on a network of professionals, which may have included psychologists, doctors, dieticians, personal trainers and others. Is being less reliant on professionals for such information an emancipatory praxis? Does ubiquitous knowledge of the self contribute to greater self-awareness in the formation of identity and persona? As Evgeny Morozov points out in the critique of self-quantifiers, self-trackers may be identifying with the means of production as opposed to the knowledge flowing from the practice of self-tracking:

It's hard to imagine the previous generations of self-trackers forming a social movement of some kind-one with its own proselytisers, regular conferences, and a set of shared goals and aspirations. The existence of such a movement would indicate that there was something cool, even laudable, about the very activity of tracking, a tracking aesthetics of sorts. As far as social movements go, this one would be all about celebrating a common means, not a common end (77).

Self-tracking and their associated cultures of technical innovation produce epistemologies of self-awareness which produce personas that cannot be divorced from the technocratic logic of "saturated" and "networked" conceptions of self which reconfigure the body relative to the self as individual, aggregated at the population level, the interior and exterior self, and the self in regards to space and time. We conclude with the assertion that although self-tracking does produce emancipatory conceptions of personhood, it does so within a defined set of parameters including oligarchy, self-representation via the proxy of mediated data of the self, regulation, asceticism and panopticism. These parameters are shaping technical affordances, interfaces, business models, routes to innovation, and policy relating to the use and re-use of personal data.

Counting steps, measuring heart rate and blood pressure daily, or tracking the quality and duration of sleep by technical means are activities that are undertaken with the objective of 
empowering one to escape the negative effects of a demanding lifestyle, and exercise freedom in decision making enabled by a direct and intimate knowledge of one's own bodily and behavioural disposition. It is not surprising then that devices like the FitBit, Jawbone UP, and a host of others are gaining universal appeal as consumer technologies aimed at the mass market. As they are marketed, such devices are portrayed as inexpensive solutions designed to help users better understand their own health. As such, they are aimed at the mass market as nongendered, catering to all levels of fitness, and technologically savvy lifestyle product:

On the walk to work, at the weight room or on the last mile. Somewhere between first tries and finish lines. Pillow fights and pushing limits. That's where you find your fitness. Every moment and every bit makes a big impact. Because fitness is the sum of your life. That's the idea Fitbit was built on - that fitness is not just about the gym. It's all the time. How you spend your day determines when you reach your goals. And seeing your progress helps you see what's possible. Seek it, crave it, live it (www.fitbit.com/whyfitbit).

However, despite the celebratory rhetoric associated with the "quantified-self movement", the self-quantifier, we argue, is unlikely to achieve authentic personal agency. We posit that the selfknowledge produced through iterative and ubiquitous quantification and documentation of one's life is distorted by various forces inherent within the industrial processes which give rise to the technology used in self-quantification, and within the various software architectures and data permissions wherein self-quantifiers generate epistemologies of self. Further, the critiques of self-quantification are masked by the ideology perpetuated in the marketing copy quoted above. By a co-opting of the Delphic philosophical tenet of 'know thyself', which constructs the self-quantifier as an emancipated figure, the self-quantifier is framed as the embodiment of the self-aware subject in the world of contemporary networked society and technocracy.

\section{LOCATING THE Politics OF SELF-TRACKING}

In regards to self-tracking, what might some of the more interesting and unprecedented political dimensions of persona formation be? In this section we discuss three emerging biopolitical trajectories which impact the ways in which self-quantification relates to the formation of a sense of self: the use of self-tracking by mandate of social or governmental institutions; the double logic of making self-tracking devices noticeable and discrete; the potential pathologies and various types of dysmorphia that are likely to emerge as self-tracking is mainstreamed.

In a blog entry on the topic of purchasing a Jawbone Up24 (\$129 - \$147 USD), Mark Carrigan, a sociologist who has been studying the Quantified Self movement, discusses how selftracking interfaces with social policy, which he describes as the "coming techno-fascism". Cardigan writes:

I'd got bored with the Nike Fuel Band, losing interest in the opaque 'fuel points' measurement and increasingly finding it to be an unwelcome presence on my wrist. I'd also been ever more aware of how weird my sleep patterns have become in the past couple of years, cycling between rising early and staying up late, with little discernible rhyme or reason. The idea of tracking my sleep in a reasonably accurate fashion, using degree of bodily movement as a cypher for the depth of sleep, appealed to me on a reflexive level. Somewhat more practically, the Jawbone's silent alarm sounded great: it gently wakes you by vibrating on your wrist at the period within a defined interval at which it detects you are in the lightest state of sleep. It's only been a few days but it really seems to work. I've woken up refreshed in a way that feels oddly natural given the rather novel consumer technology that's bringing it about. ... 
Consumer self-tracking devices and schemes like this serve to normalise tracking of this sort. What comes next? How hard is it to imagine a situation where a Conservative government, eager to separate 'strivers' from 'skivers' demands that welfare recipients submit to monitoring of their alcohol and nicotine intake?

Here we can see a strong resemblance between antecedent technologies to the current generation of self-tracking wearables such as house arrest bracelets or remote alcohol monitoring devices. Other developments can be seen in Fitbit's collaboration with designer Tory Burch, which takes the utilitarian design and function of the standard rubber Fitbit and provides an alternative collection to the consumer that is metal and made to resemble a piece of jewellery. This trend to further domesticate and differentiate the device within the existing Fitbit product range is reflected by the fact that the Burch design retails approximately $\$ 50$ USD more than the standard Flex model (\$129.95USD) indicating differentiation beyond point-forpoint comparison of products based on functionality alone. With increased opportunity to 'choose' the type of device one attaches to the body, the practice of self-tracking is normalised in everyday life, and consumers are provided the ability to outwardly display either a utilitarian or an ornamental identification with the practice. This is a radical departure from Carrigan's reflections on forced industrial and social schemes tracking, but is nonetheless an indication of how users are being catered for in the current consumer marketplace of wearable technologies. This departure also shows how such systems can be further assimilated into the performative dimension of embodying the self-tracking ideology with some, albeit limited, degree of individuality and gender specificity. This invites the question: is a jewellery-like self-tracking device designed to draw attention to the fact that one is self-tracking or suggestive of the potential to make such technologies discrete, especially when being used for the purposes of the third-party mandated monitoring individuals as Carrigan suggests.

The self-quantifier persona also carries within it a number of potential pathologies. These are self-commodification and disciplining, technology fetishisation, transference of one's attention away from organic reflection into a system of numerical representation, idiosyncratic behaviours, various hypochondria's and forms of self-medication that may have a deleterious impact on one's health. These are lines of enquiry which are yet to be seen in conversations at the quantified self meet-ups or on the various web forums linked to self-tracking communities . Addressing the potential downside of their experiments will undoubtedly be a fruitful area for future study in the coming years. It is here that we suggest persona studies can make a contribution as it engages with future presentations of the self relative to past accounts, mirroring the need to understand the devices themselves from the perspectives of technical antecedents and related devices outside the established category of "health-tracking". This contribution could see the interfacing of media archaeology (particularly useful given its emphasis on alternate genealogies of technical systems of knowledge production and representation) and persona studies around the objective of understanding the public self and its technological predispositions (Parikka; Barbour et al.).

Self-quantification practices, namely practices occurring within the named 'quantified self' movement, frame the production of self-knowledge "around" a particular category of technology: sensors, wearables, ubiquitous computing and their associated set of informational artefacts. The statistics, graphs and predictions-such as life expectancy based on resting heart rate data-resulting from self-quantification are inevitably gaining currency as authentic, unbiased mediations of self (Bijker 123). One way that the authenticity of such data may be challenged is by viewing self tracking systems as 'remediating' self-knowledge through 
networked, sensor enabled, and data driven technologies which then intersect with cultures of self-help and self-therapy (Bolter and Grusin).

The self-quantifier persona is constructed by those who wish to promote the use of selftracking devices as a strategically and 'technocratically rational' figure. However, the ideal selfquantifier persona image perpetuated by the marketing messages of Fitbit or Apple is unlikely to sense the extent to which their self-commodification is automated and internalised. $\mathrm{N}$. Katherine Hayles suggests that the unconscious nature of sensor data means that the human perception of self and environment takes place within an ontology where technical artefacts carry part of the cognitive load associated with the processing of sense data (Hayles). Embedded in an epistemological site of knowledge production distributed in a way that makes the underlying codes and software operating to produce that knowledge "non conscious." By existing below a certain cognitive threshold, yet intensifying the level of awareness of the self, self-quantification constitutes a complex, and dialectical relation of self-intimacy and selfabstraction where it becomes difficult to recognise the extent to which self-tracking commodifies the processes being mediated (Townley). In addition to this, the desire for security and anxiety reduction is articulated within the symbolism of consumer products emerging through the wearable technology and quantified self pipeline, such as Whistle an activity monitoring system for pets. Buying into cultures of self-quantification-ideologically, and transactionally-means that a visual and physical narrative of life can be constructed and then drawn upon as a resource especially in times of stress or uncertainty.

Looking at these practices historically, by the mid 1990s Microsoft Research, led by Gordon Bell, Jim Gemmell and Roger Leuder had begun to explore ways in which a 'memex' like memory extending system for assisting in the production of intimate knowledge of self could be developed (Bush). They imagined that:

If you choose, you'll be able to create this digital diary or e-memory continuously as you go about your life. This will be nearly effortless, because you'll have access to an assortment of tiny, unobtrusive cameras, microphones, location trackers, and other sensing devices that can be worn in shirt buttons, pendants, tie clips, lapel pins, brooches, watchbands, bracelet beads, hat brims, eyeglass frames, and earrings. Even more radical sensors will be available to implant inside your body, quantifying your health. Together with various other sensors embedded in the gadgets and tools you use and peppered throughout your environment, your personal sensor network will allow you to record as much or as little as you want of what happens to you and around you (Bell and Gemmell 28).

More recently, Nicholas Feltron, a former Facebook employee has collected data on various aspects of his life and authored a series of personal annual reports which represent each year of his life between 2005 to 2013 (Feltron.com). Graphing variables such as travel miles, music listened to, photographs taken, books read, food and drink consumed and so on. Each year Feltron added new metrics to the report. In 2007 for instance, he added time spent walking and time spent on busses, taxis and trains. In 2008, using openstreetmap.com, he mapped out a spatial timeline-Feltron is credited with developing the Facebook timeline-of his life detailing major events such as the election of Barack Obama, or his "favourite lunch with Dad" producing a macrosopic and subjective map of his life. In 2009, the project developed further when Feltron solicited feedback by way of an online survey of every person with whom he had "meaningful encounters" with over the course of the year, and overlayed data on conversation topics, mood and demeanour. Later iterations of the report were more sophisticated, 2010 focused on relationships, namely with his father and the 2012 report makes use of a custom 
built mobile application called "Reporter" which randomly sent reminders to Feltron to complete a survey answering the following questions: Where are you? Who are you with? What are you doing? What are you wearing? What are you eating? What are you using? What are you drinking? Are you asleep?

These experimental techniques and the understandings that they produce will undoubtedly become more commonplace as tools, deployed in the production of private and social understandings of the body and one's life-biography, altering the scale and intensity of productive capacities, as a commodity, as diseased, gendered and as 'normal'. The ability to relate these understandings to the body's materiality and dimensions (weight, height, steps), physiology (heart rate, metabolism, respiration), sensory thresholds (eyesight, hearing, pain and nociception), numerical indexing such as body mass index (BMI) and other states of being (hunger, thirst, anxiety etc.) as units for the quantitative comparison and measurement further produce bodily dimensionality relative to certain parameters. Drawing on Georges Canguilhem's notion of a habitual and ideal state, designated by the "pathological normal", Elisabeth Stephens reminds us that the normalisation of a body by set parameters that denote health and/or productivity is not only a matter of "moving subjects towards" a numerical or statistical range denoting normalcy, but also "measuring the gaps and differences by which they deviate from that norm" (Stephens).

In regard to the quantified self, participants hold a belief that:

The relationship between "habit formation" and the limitations of devices is significant. On one hand, the habits/practices that most participants sought to instill [sic] in themselves generally (though not always) adhered to normative guidelines around health and good citizenship: exercise more, work more effectively, keep moods elevated, etc. On the other hand, these clearly are not passive consumers swallowing blindly the parameters of "what's good for them." In many ways they see their activities as a response to big data and big science dictums that make claims about the healthy body from on high. In the face of generalised, anonymous one-size-fits-all prescriptions derived from population studies, they seek to understand what is right for me. What is the optimal bedtime for me? Under what diet regime do I feel my best? What activities (sleep, caffeine, wheat, dairy, and other usual suspects) are particularly correlated with mood or energy in my life? (Nafus \& Sherman)

What may result out of self-quantification is a further mutation of normalcy where "we are unable or unwilling to realise (individually or en masse) that we are becoming radically alone" in our pursuit of self-discovery. In its most extreme form, this may foster the notion that it is the internal reflection and measurement that counts, no matter how it is achieved (Hassan). The annual report or dataset becomes the instrument through which the subject constructs persona and it also becomes the informational asset in which any claim of an authentic self or persona is evidenced.

The various modes of personal data production - private, pushed (encouraged), communal, imposed or exploited-add further complexity to the self-quantifier persona (Lupton). The development of self-knowledge through these different forms of tracking, each with different motivations and perceived utility are, therefore, likely to be obscured by the context dependent factors associated with these various modes. For example, acceptance of surveillance and quantification exacerbates anxiety of not only others, as in the case of personal alarms and CCTV, but also a potential self materialised in the numbers if they go the wrong way or deviate from what is normal. This furthers the uncertainty to which one might feel they are in touch with the self or able to "listen" and intuit the body. Attaching a Fitbit to one's wrist 
produces, something that relates back to what Maurice Merleau-Ponty, writing in the 1940s, described as, an "incomprehensible twist in an organic process [of self awareness and proprioception] ... where a human act becomes torpid and is continued absent mindedly in the form of a reflex" (87). In the next section we discuss how these practices of self-quantification are intersecting the fields of lifestyle marketing, consumer technology, and discussions of health and illness.

\section{KNOWING AND HEALING THE SELF}

The production of self-knowledge then has become a valuable social, economic, and moral resource in the management of everyday life. The notion that a person can change though self-knowledge presumes that an inner locus of knowledge and experience is one that is equivalent to or subordinates the material world. This locus of knowledge and experience provides the major rationale for central institutions of democracy, the law, the state, education, community, and more importantly the notion of a self with a certain degree of agency. These all operate on tenets of enhancement of physical and mental functioning, building character and will, engaging in independent judgement, intentionality, memory, and conscious knowledge (Gergen).

Notwithstanding a long polyvalent history which includes popular psychology and the deep ties of asceticism and self-discipline to religiosity, the postmodern pop-culture popularisation of the self-help genre of books, psychometric testing is perhaps a suitable place to begin to develop a critical understanding of self-reflexivity. This self-reflexivity is achieved through proliferation of minuscule sensor technologies that can be worn or embedded somewhere in a person's home or work environment.

The self-help book phenomena rests on the assumption that self-directed reflexive analysis is required to achieve the ultimate aim of developing a personal philosophy aligned to the given objective of the text, be it the accumulation of personal wealth, achieving better health, overcoming addictions, forming more enriching relationships or any number of other life-goals. Inherent within the logic of the self-help genre is the conveying of sometimes confronting "home truths" which when recognised enable the reader to observe some personal trait that has been obscured by the anomie associated with an increasingly fast paced and complex world. It can be noted, however, that if the goal-directed nature of the text is removed then self-help books may be reduced to over determined or misappropriated folk wisdom and vacuous platitudes of self-empowerment. Another significant feature in the logic of self-help books is the invitation to reflexively narrativise one's life which includes an inventorying or diary keeping as a means to track progress, and "thinking ahead" about how one's habits and behaviours in the present may have longer term effects (Rainwater, cited in Giddens 72). For instance, a record of alcohol consumption, recreational drug use, risk-taking behaviours, or even weight fluctuations or tracking of mood, may be used as a resource to be drawn on during the self-help undertaking outlined in the book. The information could also be retained for use in the future, should the need arise and this information become relevant in some form of selfdirected lifestyle intervention or administered therapy. Further, the reader of self-help books identifies as a distinct subscriber to an ideologically loaded set of ideas outlined by the authoroften given authority as a first person account of personal transformation. For example in her bestselling book Thrive, Ariana Huffington promotes the idea of wellbeing as a "third metric", which along with money and power define success. The reading of self-help books are thus an individual undertaking, and ideological interpellation, where peer support is displaced into a broader discontiguous network of relations either within or outside the auspices of group therapy or mutual aid, that is, those reading the book. 
Given that self-directed intervention into one's own life involves risk in that it forces one to confront material realities and open possibilities, the individual must be guided by something more material than intuition and in which security can be sought to demonstrate that one's efforts at self-betterment are not in vain (Giddens 73). It is not surprising then in the decades following the popularisation of print-based self-help culture that numerous technical innovations have begun to feature in the industry for self-help and self-knowledge. Self-tracking practices combine introspective methods, experimental methods and the phenomenological methods of human centric investigation, the quantitative measures of the survey researcher, and the qualitative measures of interpretation.

Numerical representation is necessary to maintain the interplay between intimacy and abstraction, and the expansion of the upper limits of what one desires relative to their perceived needs. In light of this, it is important to note that the model for organising self-tracking communities such as the quantified self are not that of an isolated subject struggling to come to grips with the increasing ontological complexity of their life-world, but that of a collectively negotiated production of knowledge through benchmarking, the analysis of gaps (where I am now, and where do I want to be?), and the assessment of one's strengths and weaknesses. This pursuit is based on a willingness to engage in self-scrutiny and in some cases self-deprecation. Furthermore it is riddled with anxieties, as Zygmunt Bauman writes:

"Health", circumscribed by its standards (quantifiable and measurable, like bodily temperature or blood pressure) and armed with a clear distinction between "norm" and "abnormality", should in principle be free from such insatiable anxiety. Again, in principle, it should be clear what is to be done in order to reach the state of health and protect it, under what condition one may declare a person to be "in good health", or at what point of therapy one is allowed to decide that the state of health has been restored and nothing more needs to be done. Yes - in principle ...

As a matter of fact, however, the status of all norms, the norm of health included, has, under the aegis of "liquid" modernity, in a society of infinite and indefinite possibilities, been severely shaken and become fragile. What yesterday was considered normal and thus satisfactory may today be found worrying, or even pathological and calling for remedy ... ever-new states of body become legitimate reasons for medical [or other types of] intervention ... (2000: 79)

The collection and visualisation of personal data are intended to capture and evoke such "matters of concern" (Latour, 2005: 87-120) which like health and fitness, mood and happiness, sleep patterns, stress, ageing, productivity, and mental performance and memory, are all sources of life-uncertainty that would otherwise be difficult to draw attention to against the background of a modern, busy, and achievement oriented life. This is especially pertinent given the longstanding interest in healthcare expenditure and its relation to Gross Domestic Product, quality of life indexes, and calculations of life expectancy (Costa-i-Font, Courbage and McGuire). These matters could manifest in things like tracking the number of glasses of water, coffee or units of alcohol consumed in a day, keeping track of bills to pay, appointments, exercise, work productivity, or progress towards any life-goal an individual may set for themselves. Obviously such striking explications of one's successes (or shortcomings) appeal to the emotions and evoke a sense of the technological sublime and a translation of a 'clean' technological fix in the form of knowledge through data. However, the messy realities of people's lives prevail against their interventionist efforts and matters of concern, matters of fact, lived experience, and recognisable situations become bound up in the production of self-knowledge as a life-resource. The data produced through tracking exists in transitional form between the intimate and public, 
as an intermediary between the subject and a projected self-in-world, self-relative-to-others "outside" which helps achieve a sense of control through self-exposure, actualisation and a sense of ownership over personal data where one must act upon but also exchange something of value with other people (de Lange, 2013).

The quantified self movement can be viewed as part of the trajectory of self-therapy where an individual's life forms part of a "planning project" for which an individual is solely responsible, and thus the parameters around which action to redefine the self might be taken (Beck-Gernsheim; Giddens 75). The project of reflexively forming a conception of self that is malleable to self-help, and by extension receptive to self-therapy, subordinates the epistemological pursuit of self-knowledge to the identification of properties of oneself that can be fashioned into markers that denote a productive and rewarding self-directed change for the better. Speaking methodologically, the fact that self-help relies heavily on self-reports introduces a number of biases relating to situational and dispositional specificities, and other personal sensitivities which may impede the extent to which one might become self-aware in the process of self-help (Donaldson and Grant-Vallone 249).

We can see the appeal of numbers in self-help given the above echoing something that Lewis Mumford identified in Technics and Civilisation, written in the nineteen thirties. Mumford reminds us that between the fourteenth and seventeenth century a significant change in the conception of the body's relation to the environment occurred. Hierarchical conceptions of the body in space were replaced by formulations of space and time that held both as configurations of relativities and magnitudes. This change in perspective, argued Mumford, brought about a new attitude towards the body that was readily assimilated into multiple facets of everyday life. As this emphasis on numerical representation and measurement grew, "tempo became faster [and] the magnitudes became greater, conceptually, modern culture launched itself into space and gave itself over to movement" until a point where "in time-keeping, in trading [and] in fighting, men counted numbers; and finally as the habit grew, only numbers counted" (Mumford 22).

For the self-quantifier, the numbers-which stem from seemingly raw data which is meant to represent material physiological and behavioural processes-are intended to foreground certain aspects of self which are being engaged in the formation of a spatially and temporally specific construction of persona relative to a life stage, or progress towards some external goal or benchmark. The quantified self can, therefore, be thought of as persona forming through processes of numeration in an "extended present" (Nowotny). This extension of selfdirected action extends to the body, via mediation, in self-quantification where the body "of an action system rather than merely as passive object" (Giddens, 77).

\section{COMMODIFYING EPISTEMOLOGIES OF SELF}

Herbert Marcuse in One Dimensional Man recognised that industrial society necessitated its own intensification in order to project onto society the solutions through which the life it produced could be made simpler, more navigable, and less atomistic. The likely reality of body monitoring-despite all changes that have occurred to grant cognitive access to one's body and oneself, the political contestation of knowledge, or information-links the pre-sensor technology era of medical instrumentation being only in the hands of specialists to the present "clinic in the pocket" era of wearables and smartphone applications in a historical continuum. However, the groups of individuals who self-quantify do alter the terms under which this knowledge is held and contested. That is, they alter the basis of their own dominion. This is 
done, as Marcuse has argued, by "gradually replacing personal dependence on the 'objective order of things' (on economic laws, the market etc.)" (147).

Marcuse argues:

To be sure, the "objective order of things" is itself the result of domination, but it is nevertheless true that domination now generates a higher rationality that of a society which sustains its hierarchic structure while exploiting ever more efficiently the natural and mental resources, and distributing the benefits of this exploitation on an ever larger scale. The limits of this rationality, and its sinister force, appear in the progressive enslavement of man by a productive apparatus which perpetuates the struggle for existence and extends it to a total international struggle which ruins the lives of those who build and use this apparatus (147-148).

Socialisation in self-quantification and tracking is difficult to resist given its propensity to be immersive, ubiquitous, and passive. The self quantifier is not likely to feel coerced into their practice but they also have little freedom to experiment despite the larger rhetoric of experimental behaviour that is bound up in $n=1$ methodology and the hacker-space like setting of meet-ups and forums where self-quantifiers share knowledge.

The potential corresponding tightening of regulatory control over movement and actions, however, is not be taken lightly when it interferes with the ability to act on desires only to repress them in order to reproduce capital in the form of a statistical baseline of normative limits on which further discipline of others is forged. A wearable sensor additionally undermines somatic knowledge-what the body already knows-in order to prime it for selfinitiated regimes of surveillance. In some ways, the subject becomes a spectator invited to watch their own objectification but not fully participate in it.

Self-quantifiers may also be motivated to gain influence in policy outside of dictates of traditional Western, evidence based, and preventative medicine. In this case, the self-quantifier becomes an actor contesting ideas of ownership of data generation and the ability to influence health policy. The use of self-tracking devices generates a distinct "networked public" (Varnelis) that is then equipped with open, and collective sources of "evidence", stemming from the collectivisation of individual knowledge. The quantified self "community" then, at the collective level, hold this data to be a productive application of their labours, distinct from their individual pursuit of data collection, even if posted and shared across social media among a more private group of peers. The value of such data is recognised widely within the fields of evidence-based medicine, the public participation in clinical trials, and in advocating for health technology that caters towards various interest groups, often ignored or deemed non-profitable by the health technology sector.

\section{QUANTIFIED ROUTES TO PERSONIFICATION}

What kind of person is the self-quantifier? While we do not suggest the existence of a singular archetypal persona, in this section we outline some routes to knowledge through which self-quantification personas can be understood. The familiar process of auditing provides a suitable point of entry. Auditing refers to a linked set of diverse practices where observation, reflection, documentation, and analysis is used to determine the credibility of certain practices or individuals, the (re)allocation of resources and the identification of certain traits within individuals which may label them as potential "talent", or "risk" to be either cultivated or mitigated respectively. Audits "evoke a common language of aspiration" yet "also evoke anxiety and small resistances, are held to be deleterious to certain goals" (Strathern 1-2). Relatedly, the 
notion of conducting a "gap analysis" (Balm) is commonplace in many facets of everyday life, which naturalise the questions: Where am I? Where do I want to be? What is standing in my way?

When auditing acquires a social presence that precipitates beyond the institutional contexts of the workplace, educational system, clinic, or notions of accountability, it also automates and internalises a form of neoliberal self-governance which is made compelling, or at least appealing. Self-quantification therefore combines, symbolically and materially, the precepts of auditing with health-orientated lifestyle marketing and web 2.0 enabled participation in a networked form of personal growth.

The popularisation of auditing as a management strategy since the nineteen eighties is a notable antecedent to the assimilation of self-quantification into the practice of everyday life, and in particular into the workplace. Given that workplace auditing, and quality assurance frameworks (such as the ISO 90000 or Triple Bottom Line reporting), along with psychometric testing programs such as the Leadership Skills Inventory and Meyers-Briggs personality-type indicators, are commonly encountered in the workplace, it is not surprising that more intimate forms of monitoring are rarely thought of as negative measures of control which may, potentially, impinge on personal freedom. Practices of self-quantification share with the more general model of auditing the setting of objectives, the measurement of performance, monitoring and evaluation, and reporting and feedback presented in the form of 'insights' or areas requiring attention as opposed to direct reprimand (Power, 113). However, structured auditing requires a degree of standardisation and control of measures which become problematic and constraining when a goal is removed. This is particularly important given the preventative focus of producing and archiving data on the body for future use, in the event that it may play some role in addressing chronic illness, establishing baseline levels of activity for some purpose such as a personal injury claim (see Jethani and Daly), or producing a record of events for the purposes of documentation or proof in some other future circumstance.

The notion of auditing, and relatedly archiving, satisfies the need for an individual to appear transparent, honest or compliant in some way and to prove to others (and oneself) that they embody certain context-dependent traits that are deemed to be desirable. Practices of selfquantification and self-surveillance create new "archival conditions" that both resist - through passivity and ubiquity of sensors, for instance, as they are used within a mainstream consumer product like the Apple Watch, and transform the "archival impulse" to store information for future use (Røssaak). As Røssaak reminds us, four mutually constitutive processes dictate the formation of personal archives, storage, preservation, classification, and access. While selfquantification discourse posits that archives produced through self-tracking are "living", dynamic, real-time forms of archiving, there are the distinct problems of data interoperability and obsolescence which renders the self-quantifiers archive as fragmented and discontiguous across a number of devices, data storage locations and file formats. This could be at the level of devices, but also at the level of the various communities and platforms that emerge around them. - Further, archives of self-quantification are kept apart by the permissions granted to users and third parties as to how their data may be retrieved and repurposed. For example, Apple specifies the following in its developer guidelines for its recent entry into the health tracking market with its health application and developer platform:

Because health data can be sensitive, HealthKit grants users control over their data by providing fine grained control over the information that the apps can share. The user must explicitly grant each app permission to read and write data to the HealthKit store. Users can grant or deny permission separately for each type of data. For example, a user could let your app read the step count 
data but prevent it from reading the blood glucose level. To prevent possible information leaks, an app does not know whether it has been denied permission to read data. From the app's point of view, if the app has been denied permission to read the data, no data type exists.

HealthKit data is not saved to iCloud or synched across multiple devices. The data is only kept locally on the user's device. For security, the HealthKit store is encrypted when the device is not unlocked (developer.apple.com).

At the level of a single individual an archive produced in this fashion is discontiguous by virtue of the parameters that one chooses to monitor and by devices that become obsolete and applications that cease to exist. At the aggregate level the archive is, at its base, structurally discontiguous. As Barbour et al., argue:

All of us know what it is like to act in a role, to wear a uniform or costume, to create a profile. More than a few of us know what it is to suffer through the 'individualising' categories of a social networking sign-up survey that do not adequately account for distinctions. Persona is all these things, or rather, through the various everyday activities of our work, social, and online selves we contribute to the accretion of the identity at the base of its structure. Persona functions like the construct or automated script that we assemble to interact with the world with on our behalf. This involves the technologies of computation and mediation and their interfaces that function to automate, produce and filter communication with us; email, blogs, Twitter accounts, and so on. These golems interconnect and can interact on their own in unpredictable ways on our behalf; connecting our Facebook account to a product, brand or petition; using Google as a portal to login into other web enabled services; or authorising an app to record our location. Then there are the traces that we leave scattered across digital networks, intranets, hard drives, and lost USB memory sticks, from scattered collections of digital photos to the contact lists of our mobile devices and the 'achievements' in our online gaming profiles. Persona can also be something that happens to us, as friends tag unflattering images via Facebook, or another Twitter user publicly addresses us with a unwanted, or unwarranted commentary, using the '@' and the '\#' functions (Barbour et. al,).

If the individualising aspects of personal metrics are coming to bear in the formation of persona as an "automated script" their function as non-person personas raises the issue of the reconstruction of persona from the archive. The application of such archives in cases of amnesia and degenerative neurological conditions are one such example where we can go beyond technological obsolescence and consider the relation of "traces" to persona.

A large group of people cultivating a persona through "common means" invites a questioning of the relationship between data and self, especially given the difficulty of embodying means that quantify health, mood, or productivity in ways that others within and/or outside the movement would see as authentic data. As Jamie Sherman, reporting from the 2012 International Quantified Self Meeting held in Amsterdam notes, views are inconclusive; some considered data being produced to indeed be a true reflection of the self, others considered data to be a partial or "murky" reflection. Others still considered data to be constantly evolving-a disembodied type of self that evolves under the volition of forces not entirely of the material body. A further position acknowledges the fragmentary nature of data which disassembles the body for it to be reconstituted relative to some objective, or that the self is not singular, but multifarious, the self is not data, but data is just one of many selves (http://epicpeople.org/how-theory-matters/). 
In order for the personification of self-quantification to occur, it needs to be first anthropomorphised: the sensor data and by extension information produced by the various applications being used needs to have meaning assigned to it. As Luciano Floridi notes:

[The] giving of meaning to, and making sense of reality (semanticisation of Being), or reaction of the Self to non-Self ... consists in the inheritance and further elaboration, maintenance, and refinement of factual narratives: personal identity, ordinary experience, community ethos, family values, scientific theories, common-sense-constituting beliefs, and so forth. These are logically and contextually, and hence sometimes fully, constrained and constantly challenged both by the data that they need to accommodate and explain and by the reasons why they were developed. Ideally the evolution of this process tends towards an ever changing, richer and robust framing of [personhood] (7-8).

Out of this tendency, Floridi identifies four trajectories relevant to understanding how sensor data is anthropomorphised to produce a data intensive construction of persona. First is the numerical representation of narratives whereby the self is compelled to produce and store data afforded by the increased capacity to self-surveil everyday life. Secondly, there is the delimitation of culture, where conceptual narratives of self are self-designed with increasingly sophisticated tools, therefore meaningful realities become exteriorised into a "community" of non-challenging and reassuring narratives loosely aligning to the quantified self ethos. Thirdly, the de-physicalisation or abstraction of reality through mediations of self/identity through notions of gender, job, and so on can all be 'framed within virtual mediation, and hence acquire an informational aura. Finally, the fourth way in which persona is abstracted is through an embodiment of the "conceptual environment" produced by the values, ideas, trends and the "intentionally privileged macro-narrative" of reducing the body and its processes to numbers, and constructing numbers as the target or locus of direct action that underpins the lexis and praxis of self-quantification (Floridi, 8-9).

An important question to ask, then, is at which point does the data gathered become actionable information and translate to knowledge of oneself? Consider the two sources of data as firstly generated and collected by any given wearable device, and the secondary input and user-curated information that is designed to give the primary data context and meaning (for example specifying to the device that half an hour was spent cycling on a morning commute, or that certain foods were consumed at dinner, which amounted to seven hundred calories). The device categorises and processes data discretely, but relative to pre-figured ranges. The data is presented back to the user via a dashboard of information, graphed and labelled, to be read. This is understood as increasing awareness of the most basic life activities for the able bodied: eating, sleeping and movement, or calorie intake, sleep patterns and physical exertion. In this way, that interventionist nature of upkeep differs from data flow between fixed and mobile sensor infrastructure, directly sensed data, and user inputted information.

Importantly, the information produced by the Fitbit and Jawbone UP, for example, are presented back to the user by an on screen dashboard that has a pronounced "community"' tab, making this data two dimensional, temporal and networked. Here, graphs, user generated photos, statistical analyses, and social interactions are served as "archive support" for memory and recall (Stiegler).

Moreover, the data collected is done so in the context of the subjective mind and body, an activity, a feeling or a mood ("I am cycling and feeling great"), and this context is unregistered and undetectable without input from the user. This examination of user interaction with such wearable devices poses several complexities in relation to the modes of 
collection, creation, and curation. Far from a construction of an identity from typical modes of internet usage and the data trails, circuits, and networks we leave throughout the course of our digital lives, wearable technologies mandate active participation, and are not implanted or incidental modes of data generation.

\section{CONCLUSION}

The use of self-quantification technologies does not resolve the issue or deal directly with the driving force behind the diversion of persona-forming knowledges towards an agenda of neoliberal achievement and self-management, but it does hold the promise of recognising the role of the lifestyle-as-commodity and lifestyle-querying technologies as a force that potentially impedes the formation of authentic self-awareness. Put differently, problems and frustrations with self-quantification technologys' ability to meet user expectation could mean that they are recognised as part of the problem rather than a solution to the problem of authentically identifying - and being taken seriously — as a self-quantifying figure and socially self-aware persona within a fickle culture of self-directed intervention and self-optimisation. As David Rothenberg in Hand's End: Technology and the limits of nature points out:

the problem is that technological installation of human presence in the world invariably involves a series of choices. Choosing particular avenues of action necessarily closes others. As technical decisions are made, the original intention is fast channeled towards those possibilities which the technology admits. The more complex and encompassing the tool, the more it implies a unique, peculiar way of thought. This is why... successful programming of a computer leads us to imagine that our own mind works the same way...Clearly technique is not merely a means: just when we think the problem is solved, the machine reveals new troubles and possibilities alike. Technology wraps us up in its circle. This cycling path represents what it means to be human in all kinds contact with the external world based on the dream of rational, planned order. Does any single external plan guide the wish? Theodor Adorno writes that techniques can "speak in a way which has nothing to do with the deliberate communication of a human message ... What looks like reification is actually a groping for the latent language of things (xv)

The dominant voices of self-quantification lead us to believe that by tracking we may be living the best, most informed life possible. But how is failure built into this? Perhaps by working harder, building more robust data, as thin datasets mark a sign of failure, that is to say "take up the cause and embody it fully" for the personification of self-quantification to be deemed "authentic". Others may not be prepared to be so dedicated to their quest for the numbers and may reflect on the trade-offs being made, but that is what makes the ideal quantifier stand above the rest.

\section{WORKS CITED}

Balm, Gerald J. "Benchmarking and gap analysis: what is the next milestone?." Benchmarking for Quality Management \& Technology 3.4 (1996): 28-33.

Barbour, Kim, P. David Marshall, and Christopher Moore. "Persona to Persona Studies." M/C Journal 17.3 (Jun. 2014). 19 Feb. 2015 <http://journal.mediaculture.org.au/index.php/mcjournal/article/view/841>. 
Bauman, Zygmunt. Liquid Modernity. Cambridge, UK: Polity Press, 2000. Print.

Beck-Gernsheim, Elisabeth. Life As a Planning Project. in Lash, Scott, and Bronislaw Szerszynski (eds.). Risk, Environment and Modernity: Towards a New Ecology. London Sage Publ, 1996. Print.

Bell, C G, Jim Gemmell, and C G. Bell. Your Life, Uploaded: The Digital Way to Better Memory, Health, and Productivity. New York: Plume, 2010. Print.

Bijker, Wiebe E. Of Bicycles, Bakelites, and Bulbs: Toward a Theory of Sociotechnical Change. Cambridge, Mass: MIT Press, 1995. Print.

Bolter, J D, and Richard Grusin. Remediation: Undestanding New Media. London: The MIT press, 2002. Print.

Bush, Vannevar. "As We May Think." Atlantic. 176.1 (1945). Print.

Canguilhem, Georges. The Normal and the Pathological. New York: Zone Books, 1989. Print.

Costa-i-Font, Joan, Christophe Courbage, and Alistair McGuire. The Economics of New Health Technologies: Incentives, Organization, and Financing. Oxford: Oxford University Press, 2009. Print.

Donaldson, Stewart I., and Elisa J. Grant-Vallone. "Understanding self-report bias in organizational behavior research." Journal of Business and Psychology 17.2 (2002): 245-260.

Gergen, Kenneth J. The Saturated Self: Dilemmas of Identity in Contemporary Life. New York: Basic Books, 1991. Print.

Floridi, Luciano. The Philosophy of Information. Oxford England: Oxford University Press, 2011. Print.

Giddens, Anthony. Modernity and Self-Identity: Self and Society in the Late Modern Age. Stanford, Calif: Stanford University Press, 1991. Print.

Golko, A.J., M.W. Schmidt, and F. Alvarez. "Wrist-Worn Electronic Device and Methods Therefor." Google Patents, 2012. Print.

Jethani, Suneel and Daly, Angela. Fitness Tracking Data in Courts - Persuasive But Not Conclusive. The Conversation 24 November 2014.

Hassan, Robert. "Our Post-Modern Vanity: The Cult of Efficiency and the Regress to the Boundary of the Animal World." Philosophy \& Technology (2014): 1-19. Print.

Hayles, N. K. (2006). Traumas of code. Critical Inquiry, 33(1), 136-157.

Huffington, Arianna S. Thrive: The Third Metric to Redefining Success and Creating a Life of Well- Being, Wisdom, and Wonder. , 2014. Print.

Latour, Bruno. Reassembling the Social: An Introduction to Actor-Network-Theory. Oxford: Oxford university press, 2007. Print.

Lefebvre, Henri. Rhythmanalysis: Space, Time, and Everyday Life. London: Continuum, 2008. Print.

Lupton, Deborah. Self-Tracking Modes: Reflexive Self-Monitoring and Data Practices (August 19) 2014. Available at SSRN: http://ssrn.com/abstract $=2483549$ or http://dx.doi.org/10.2139/ssrn.2483549

Marcuse, Herbert. One-dimensional Man: Studies in the Ideology of Advanced Industrial Society. Boston: Beacon Press, 1964. Print.

McLuhan, Marshall. Understanding Media: The Extensions of Man. , 1964. Print.

Merleau-Ponty, Maurice. Phenomenology of Perception. Oxon: Routledge, 2014. Print.

Morozov, Evgeny. To Save Everything, Click Here: The Folly of Technological Solutionism. , 2013. Print.

Mumford, Lewis. Technics and Civilisation. New York N.Y.: Harcourt, Brace and Co, 1934. Print.

Nafus, Dawn, Sherman, Jamie. "The Quantified Self Movement is not a Kleenex." <http://blog.castac.org/2013/03/the-quantified-self-movement-is-not-a-kleenex/>. Accessed 10 December 2014.

Nowotny, Helga. Time: The Modern and Postmodern Experience. Cambridge, UK: Polity Press, 1994. Print.

Power, Michael. "The audit society—Second thoughts." International Journal of Auditing 4.1 (2000): 111-119. 
Røssaak, Eivind. "The Archive in Motion: An Introduction." The Archive in Motion (2010): 1126.

Rothenberg, David. Hand's End: Technology and the Limits of Nature. Berkeley: University of California Press, 1993. Print.

Strathern, Marilyn. Audit Cultures : Anthropological Studies in Accountability, Ethics, and the Academy. London; New York: Routledge, 2000. Print.

Stephens, Elizabeth, "Normal, normalisation, normativity", Transgender Studies Quarterly, vol. 1, no. 1 2013, pp. 203-206.

Stiegler Bernard, and Stephen Barker. Technics and Time. 2, Disorientation. Stanford, Calif.: Stanford University Press, 2009. Print.

Stivoric, J., F. Gemperle, and C. Kasabach. "Wearable Human Physiological Data Sensors and Reporting System Therefor." Google Patents, 2003. Print.

Townley, Barbara. Reason's Neglect: Rationality and Organising. Oxford: Oxford University Press, 2008. Print.

Wicklund, Robert A, and Martina Eckert. The Self-Knower: A Hero Under Control. New York: Plenum Press, 1992. Print.

Varnelis, Kazys. Networked Publics. Cambridge, Mass: MIT Press, 2008. Print.

Wolf, Gary. "The data-driven life." The New York Times 28 April 2010.

Yuen, S.G.J., J. Park, and E.N. Friedman. "Portable Monitoring Devices and Methods of Operating Same." Google Patents, 2012. Print.

Suneel Jethani is a Ph.D. candidate and lecturer in the School of Culture and Communication at the University of Melbourne, Australia.

Nadine Raydan is a PhD candidate in the College of Design and Social Context at the Royal Melbourne Institute of Technology 